Classification

Physics Abstracts

$61.30-64.70 \mathrm{M}$

\title{
Pincements de Skoulios-de Gennes : observations numériques
}

\author{
C. Godrèche et L. de Seze \\ Service de Physique du Solide et de Résonance Magnétique, \\ CEN-Saclay, 91191 Gif-sur-Yvette Cedex, France
}

(Reçu le 12 septembre 1984, accepté le 12 novembre 1984)

\begin{abstract}
Résumé. - Nous examinons la version discrète d'un modèle proposé par de Gennes pour décrire la structure colonnaire à température nulle de molécules discotiques. Les observations numériques confirment la présence de " pincements » dans l'état fondamental et précisent leur structure.
\end{abstract}

Abstract. - We study the discrete version of a model proposed by de Gennes to describe the columnar
arrangements, at zero temperature, of plate-like molecules. Numerical observations confirm the
presence of pinched regions in the ground state and give a more precise description of their structure.

\section{Modèle.}

1.1 InTRODUCTION. - L'observation aux rayons $\mathrm{X}$ d'empilements discotiques de phtalocyanines semble indiquer l'existence d'une distorsion périodique des colonnes $[1,2]$. De Gennes a proposé un modèle simple pour l'étude structurale de ces systèmes [3]. Dans la limite continue du modèle, il conjecture pour l'état fondamental la présence de "pincements " qui seraient à l'origine de la distorsion périodique observée. Nous reprenons ici l'étude de ce modèle, dans sa version discrète, par des méthodes numériques.

Le système étudié est schématisé sur la figure 1. Le centre de gravité des plateaux est localisé en $x_{i}$. celui des chaînes en $y_{i}$. Le modèle est décrit par l'énergie :

$$
\phi=\sum_{i} U\left(x_{i+1}-x_{i}\right)+\frac{A}{2}\left(y_{i+1}-y_{i}-b\right)^{2}+\frac{C}{2}\left(x_{i}-y_{i}\right)^{2} .
$$

Les trois termes qui interviennent dans l'énergie décrivent respectivement l'interaction entre les plateaux (de type Lennard-Jones, cf. Fig. 2), l'interaction entre chaînes, d'espacement naturel $b$, enfin l'attache plateau-chaîne. La détermination de la structure à température nulle consiste à minimiser l'énergie (1) par rapport aux positions $x_{i}, y_{i}$.

1.2 EquATIONS DE MINIMISATION. - La dérivation de $\phi$ par rapport aux variables $x_{i}$ et $y_{i}$ donne les équations :

$$
\begin{array}{r}
U^{\prime}\left(\delta_{i}\right)-U^{\prime}\left(\delta_{i+1}\right)+C\left(x_{i}-y_{i}\right)=0 \\
A\left(\Delta_{i}-\Delta_{i+1}\right)+C\left(y_{i}-x_{i}\right)=0
\end{array}
$$




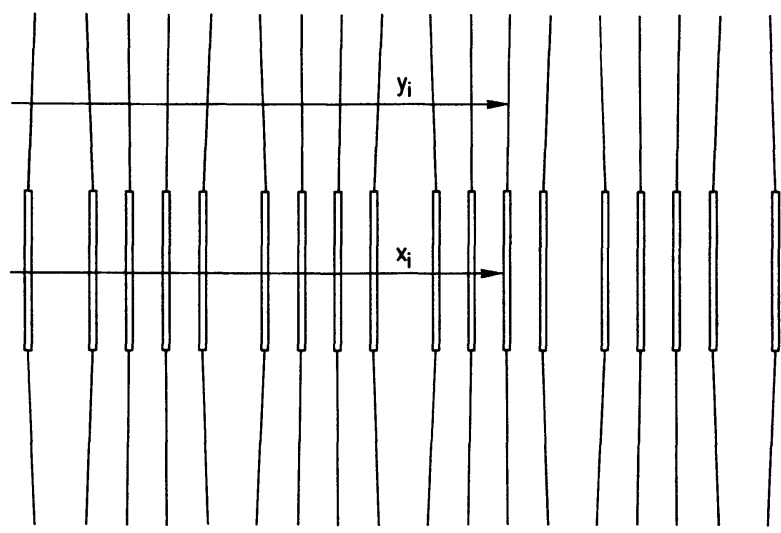

(a)

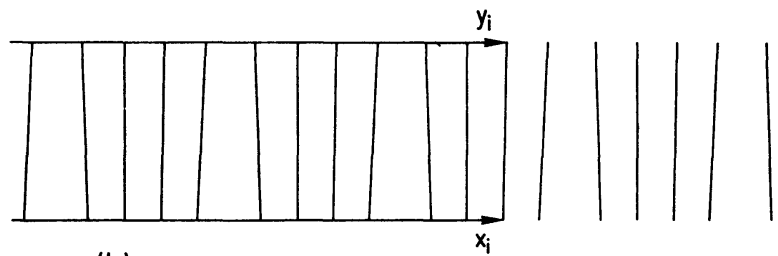

(b)

Fig. 1. - (a) Empilement discotique. (b) Représentation schématique de l'empilement.

[(a) Stacked molecules inside one column of a discotic liquid crystal. (b) Schematic representation of (a).]

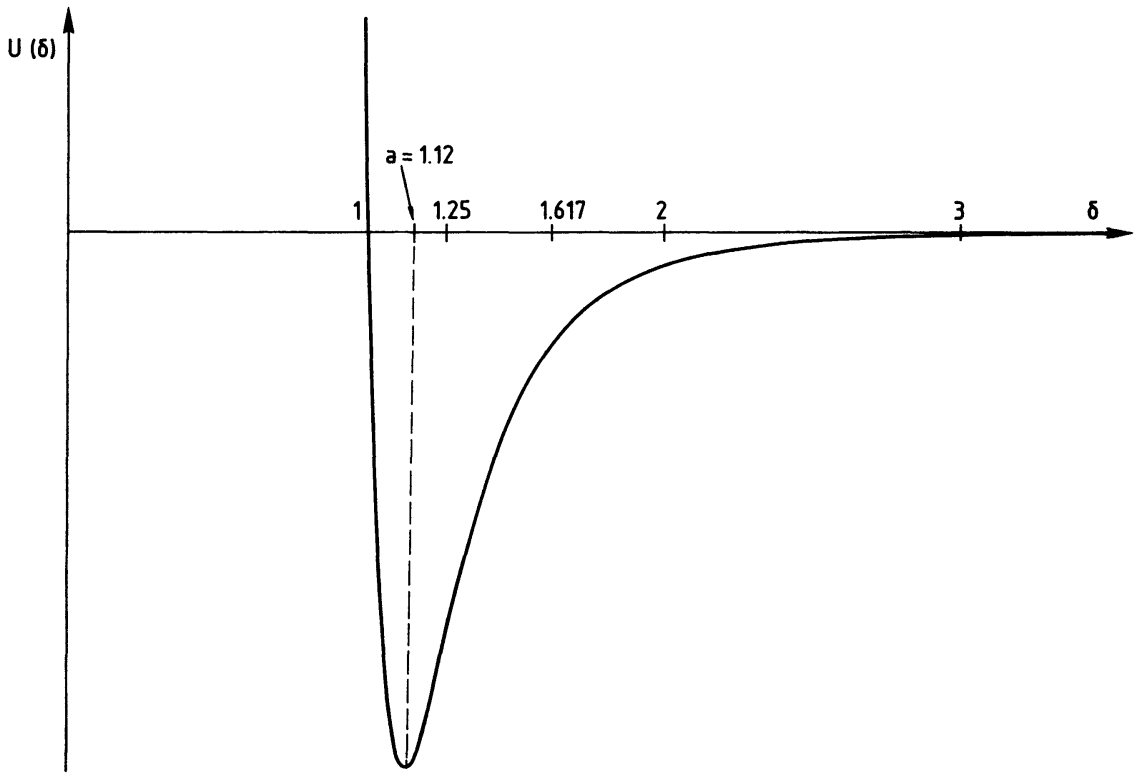

Fig. 2. - Potentiel de Lennard-Jones $U(\delta)=\delta^{-12}-\delta^{-6}$. Le minimum est atteint pour $\delta=a=2^{1 / 6}$. [Lennard-Jones potential $U(\delta)=\delta^{-12}-\delta^{-6}$. The minimum is reached when $\delta=a=2^{1 / 6}$.] 
où $\delta_{i}=x_{i}-x_{i-1}$ et $\Delta_{i}=y_{i}-y_{i-1}$. On en déduit aisément les relations :

$$
\begin{aligned}
\delta_{i} & =\Delta_{i}+\frac{1}{C}\left\{U^{\prime}\left(\delta_{i+1}\right)+U^{\prime}\left(\delta_{i-1}\right)-2 U^{\prime}\left(\delta_{i}\right)\right\} \\
\Delta_{i+1}-\Delta_{i} & =\frac{1}{A}\left\{U^{\prime}\left(\delta_{i+1}\right)-U^{\prime}\left(\delta_{i}\right)\right\} .
\end{aligned}
$$

Tenant compte des conditions de bords libres ou des conditions périodiques, l'équation (5) conduit à :

$$
\Delta_{i}=b-\frac{1}{A} U^{\prime}\left(\delta_{i}\right)
$$

Remarque : dans le cas particulier de la solution banale où tous les plateaux sont équidistants (et donc les chaînes alignées avec ceux-ci) l'énergie par maille est

$$
\phi=U(\delta)+\frac{A}{2}(\delta-b)^{2} .
$$

En minimisant cette énergie par rapport à $\delta$, on retrouve l'équation (6) dans le cas particulier $\delta_{i}=\Delta_{i}(i=1,2, \ldots):$

$$
\delta=b-\frac{1}{A} U^{\prime}(\delta)
$$

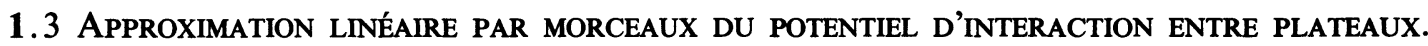
MODÈLE DE SPINS. - Il est possible de montrer, d'une façon très simplifiée, l'existence de pincements et le mécanisme de leur apparition quand $b$, distance naturelle entre les chaînes, varie. Nous prenons l'approximation suivante pour le potentiel $U(\delta)($ Fig. 3) :

$$
\begin{aligned}
U(\delta) & =\lambda\left(\delta-a_{2}\right) & & a_{1}<\delta<a_{2} \\
& =0 & & a_{2}<\delta .
\end{aligned}
$$

Dans la région $\delta<a_{1}, U(\delta)$ est une fonction décroissante que nous n'avons pas besoin de préciser car, si $b>a_{1}$, cette région n'est pas visitée par les coordonnées du modèle.

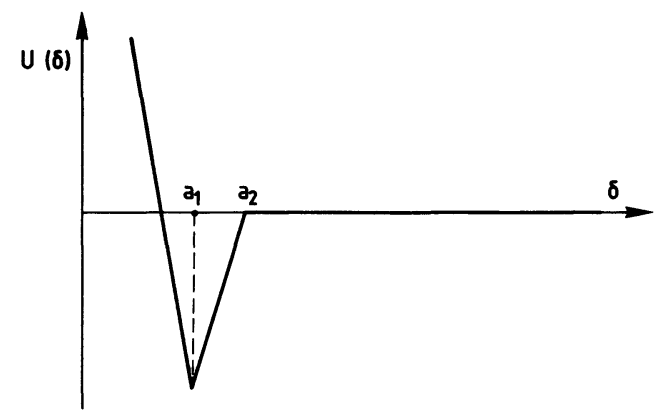

Fig. 3. - Approximation linéaire par morceaux du potentiel de Lennard-Jones.

[Piecewise linear approximation of the Lennard-Jones potential.] 
Nous introduisons le pseudo-spin $\sigma(\delta)$ tel que :

$$
\begin{aligned}
\sigma(\delta) & =-1 & & \delta_{i}<a_{2} \\
& =1 & & \delta_{i}>a_{2} .
\end{aligned}
$$

On peut alors réécrire le potentiel $U(\delta)$ dans la région $\delta>a_{1}$ sous la forme :

$$
U(\delta)=\frac{1-\sigma(\delta)}{2} \lambda\left(\delta-a_{2}\right) .
$$

En utilisant les équations (2) à (5) et en notant $\sigma\left(\delta_{i}\right)=\sigma_{i}$ on obtient l'expression des distances en terme de spins :

$$
\begin{aligned}
\Delta_{i} & =b+\frac{\lambda}{2 A}\left(\sigma_{i}-1\right) \\
\delta_{i} & =\Delta_{i}+\frac{\lambda}{2 C}\left(2 \sigma_{i}-\sigma_{i+1}-\sigma_{i-1}\right) \\
x_{i}-y_{i} & =\frac{\lambda}{2 C}\left(\sigma_{i}-\sigma_{i+1}\right) .
\end{aligned}
$$

Ces équations permettent de transformer l'expression de l'énergie. On obtient par substitution :

$$
\phi=\sum_{i}\left[J \sigma_{i} \sigma_{i+1}-H(b) \sigma_{i}\right]-J+H(b)
$$

avec

$$
J=\frac{\lambda^{2}}{4 C}, \quad H(b)=\frac{\lambda}{2}\left(b-a_{2}\right)-\frac{\lambda^{2}}{4 A} .
$$

Le modèle initial est donc, avec cette approximation linéaire au potentiel $U(\delta)$, ramené à celui d'une chaîne d'Ising avec interaction antiferromagnétique.

La structure de l'état fondamental s'en déduit aisément :

(i) $b<b_{1}=a_{2}+\frac{\lambda}{2 A}-\frac{\lambda}{C}$. Structure banale :

$$
\downarrow \downarrow \downarrow \downarrow \quad \sigma_{i}=-1, \quad \delta_{i}=\Delta_{i}=b-\frac{\lambda}{A} \quad(i=1,2, \ldots) .
$$

La période est $d(b)=b-\frac{\lambda}{A}$.

(ii) $b_{1}<b<b_{2}=a_{2}+\frac{\lambda}{2 A}+\frac{\lambda}{C}$. Structure pincée à 2 molécules ou antiferromagnétique (Fig. 4) :

$$
\begin{aligned}
\uparrow \downarrow \uparrow \downarrow & \sigma_{i}=1, & \delta_{i}=b+\frac{2 \lambda}{C}, & \Delta_{i}=b \\
\sigma_{i} & =-1, & \delta_{i}=b-\frac{\lambda}{A}-\frac{2 \lambda}{C}, & \Delta_{i}=b-\frac{\lambda}{A} .
\end{aligned}
$$

La période est $d(b)=2 b-\frac{\lambda}{A}$. 


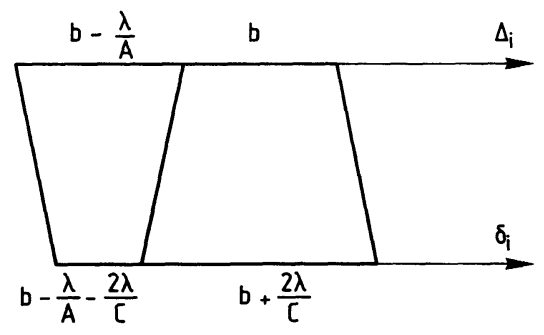

Fig. 4. - Structure pincée à 2 molécules.

[Pinched structure with 2 molecules.]

(iii) $b_{2}<b$. Structure banale :

$$
\uparrow \uparrow \uparrow \uparrow \quad \sigma_{i}=1, \quad \delta_{i}=\Delta_{i}=b \quad(i=1,2, \ldots) .
$$

La période est $d(b)=b$.

La figure 5(a) donne l'allure de la période par molécule $\frac{d(b)}{N}(N=1$ pour les structures banales, $N=2$ pour la structure pincée). Le diagramme de stabilité de la structure se lit sur la figure 5(b).

Les caractéristiques précédentes, bien que caricaturales, sont présentes dans les résultats obtenus par les méthodes numériques qui suivent. En particulier on trouve pour les petites et grandes valeurs du paramètre $b$ la structure banale et, dans la région intermédiaire, des pincements.

\section{Etude numérique.}

Pour les calculs nous avons utilisé la forme suivante de potentiel

$$
U(\delta)=\delta^{-12}-\delta^{-6},
$$

et fixé les constantes $A$ et $C$ intervenant dans l'équation (1) à 1 .

La recherche du minimum de $\phi$ a été effectuée numériquement par une méthode dite "de gradient $»$ qui consiste à simuler une dynamique fictive :

$$
\begin{aligned}
& \frac{\mathrm{d} x_{i}}{\mathrm{~d} t}=-\frac{\partial \phi}{\partial x_{i}} \\
& \frac{\mathrm{d} y_{i}}{\mathrm{~d} t}=-\frac{\partial \phi}{\partial y_{i}}
\end{aligned}
$$

Dans la mesure où nous nous intéressons uniquement aux états d'énergie minimale, l'intégration de ces équations a été faite par une méthode du $1^{\mathrm{er}}$ ordre avec un pas en temps $\delta t$ aussi grand que possible compte tenu des problèmes de stabilité numérique :

$$
\begin{aligned}
& x_{i}(t+\delta t)=x_{i}(t)-\delta t \frac{\partial \phi}{\partial x_{i}}(t) \\
& y_{i}(t+\delta t)=y_{i}(t)-\delta t \frac{\partial \phi}{\partial y_{i}}(t)
\end{aligned}
$$

Dans un premier temps nous avons étudié des chaînes relativement longues (40 à 100 molé- 


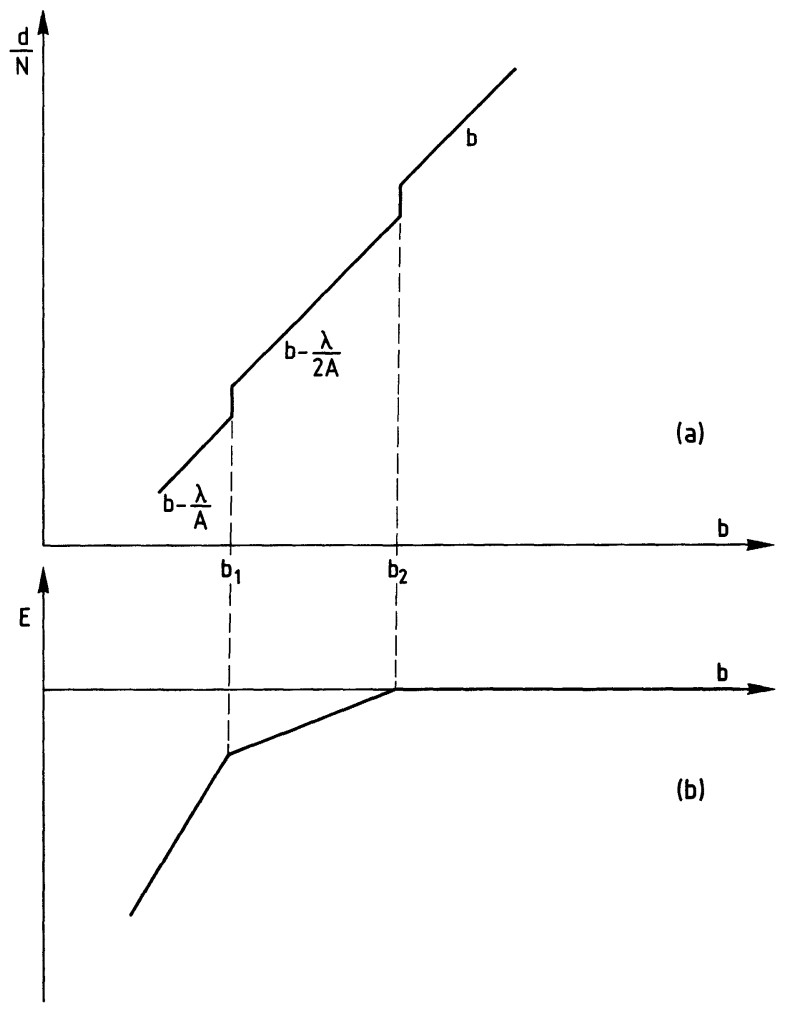

Fig. 5. - (a) Distance moyenne entre molécules. (b) Energie moyenne par molécules dans l'état fondamental $\left(b_{1}\right.$ et $b_{2}$ sont définis dans le texte).

[(a) Mean intermolecular distance. (b) Mean energy per molecule in the ground state $\left(b_{1}\right.$ and $b_{2}$ are defined in the text).]

cules) en partant de configurations initiales au hasard. Nous avons ainsi obtenu des états métastables dépendant fortement de l'état initial et du pas en temps $\delta t$.

Selon la valeur de $b$ ces états sont soit une suite de pincements à 2, 3 ou 4 molécules (Fig. 6), soit la configuration banale sans pincement.

Nous avons donc dans un second temps étudié des solutions périodiques de pincements à $N$ plateaux pour des valeurs de $N$ allant de 1 (solution banale) à 20 . Nous avons ainsi obtenu le diagramme de phase de la figure 7 ainsi que la variation de la période moyenne par molécule quand $b$ varie (Fig. 8). Quand on part des grands $b$ la structure d'énergie minimale est d'abord

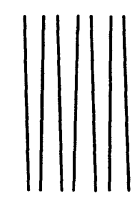

$N=2$

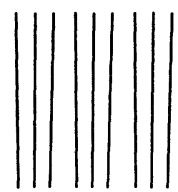

$\mathrm{N}=3$

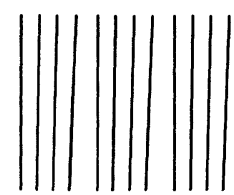

$\mathrm{N}=4$

Fig. 6. - Pincements à 2, 3, 4 molécules.

[Pinched regions with 2, 3, 4 molecules.] 

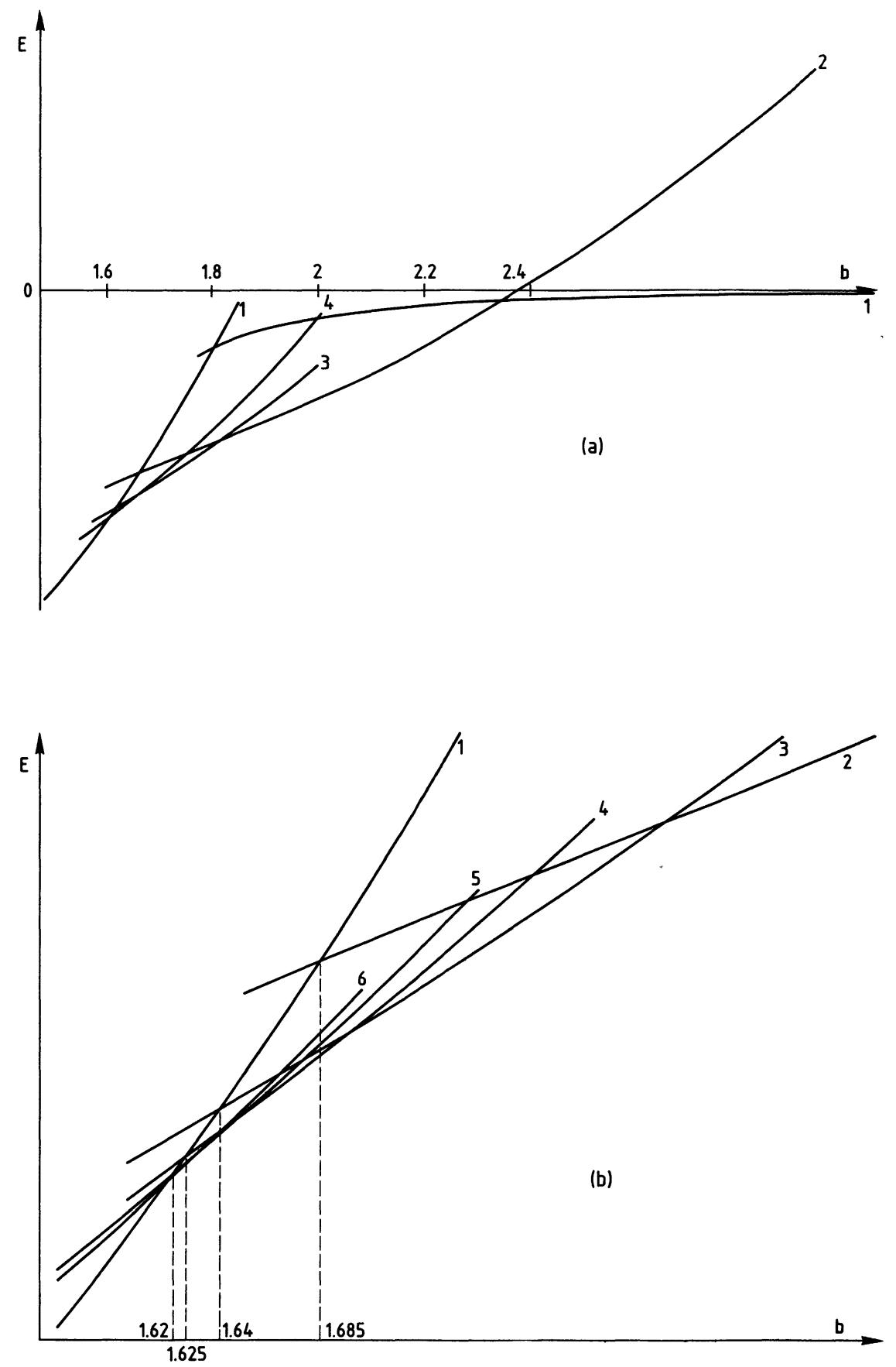

Fig. 7. - (a) Energie moyenne par molécule de configurations avec pincements à 2, 3, 4, 5, 6 molécules. (La solution banale sans modulation est notée 1.) (b) Détail du diagramme $E(b)$ de la figure $7 \mathrm{a}$.

[(a) Mean energy per molecule for pinched structures with 2, 3, 4, 5, 6 molecules (the trivial structure with no modulation is denoted 1). (b) A detail of figure 7a.] 


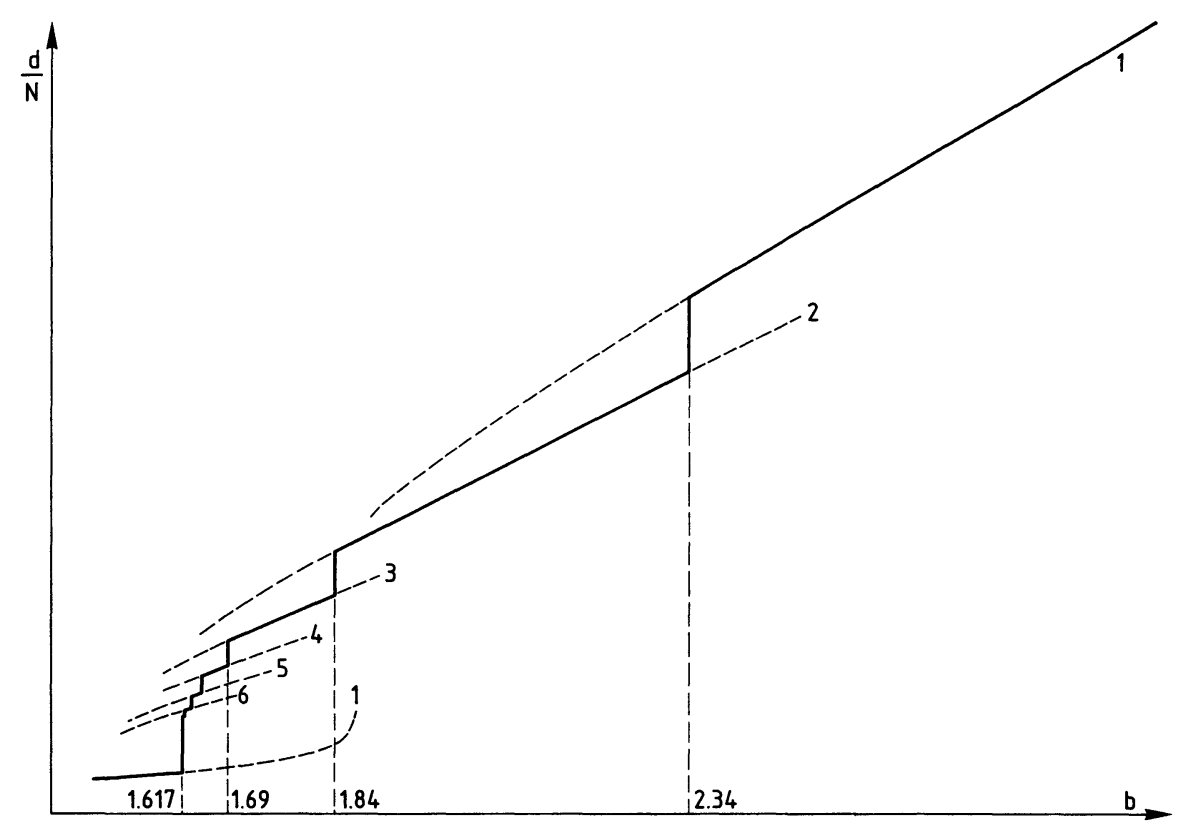

Fig. 8. - Distance moyenne entre molécules.

[Mean intermolecular distance.]

la structure banale sans pincement (notée 1 sur la figure 7) puis quand $b$ décroît, la structure pincée à 2 molécules, 3 molécules.... Pour $b<b_{1}=1.617$... on retrouve la structure banale. Le point $b_{1}$ est un point d'accumulation; quand $b$ tend vers $b_{1}$ par valeurs décroissantes le nombre $N$ de molécules dans le pincement tend vers l'infini. En d'autres termes la densité des pincements tend vers zéro. Il faut noter que quand $N$ tend vers l'infini, l'écart entre deux plateaux au pincement tend vers une limite $\delta_{\infty}$ et les écarts $\delta_{i}\left(\right.$ et $\left.\Delta_{i}\right)$ loin du pincement tendent vers la valeur $\delta$ de la structure banale. La figure 9 donne l'allure du pincement à $N=20$ molécules, valeur pour laquelle le régime asymptotique est déjà atteint.

La méthode numérique décrite ci-dessus ne permet pas d'obtenir l'état d'énergie minimale mais des états métastables (ou minima locaux). Nous avons donc cherché des états d'énergie plus basse que celle des solutions périodiques.

Nous avons d'abord étudié des mélanges de pincements à nombre de molécules différents. Les solutions obtenues ont toujours été moins favorables que les solutions périodiques.

Nous avons alors essayé, sur une suggestion de S. Aubry, une méthode fondamentalement différente qui s'apparente aux méthodes de transfert utilisées en mécanique statistique. On considère une chaîne unidimensionnelle dont les atomes peuvent être dans $S$ états discrets. Supposons connues les énergies minimales $E_{N}(s)$ de la chaîne de $N$ atomes, où le $N^{\text {ème }}$ atome est dans l'état $s$ $(s=1, \ldots, S)$ et le premier dans un quelconque des $S$ états.

On peut alors déterminer $E_{N+1}(s)$ en choisissant pour chacun des $S$ états du $N+1^{\text {ème }}$ atome l'état du $N^{\text {ème }}$ atome qui minimise l'énergie totale. Si on garde trace des choix successifs $(N=1, \ldots, M)$, partant des $S$ états du $M^{\text {ème }}$ atome, on peut remonter la séquence. Quel que soit l'état de départ choisi pour le $M^{\text {ème }}$ atome, après quelques pas, caractéristiques d'une longueur de cohérence de la chaîne, tous les chemins convergent vers un chemin unique qui est l'état fondamental de la chaîne. 


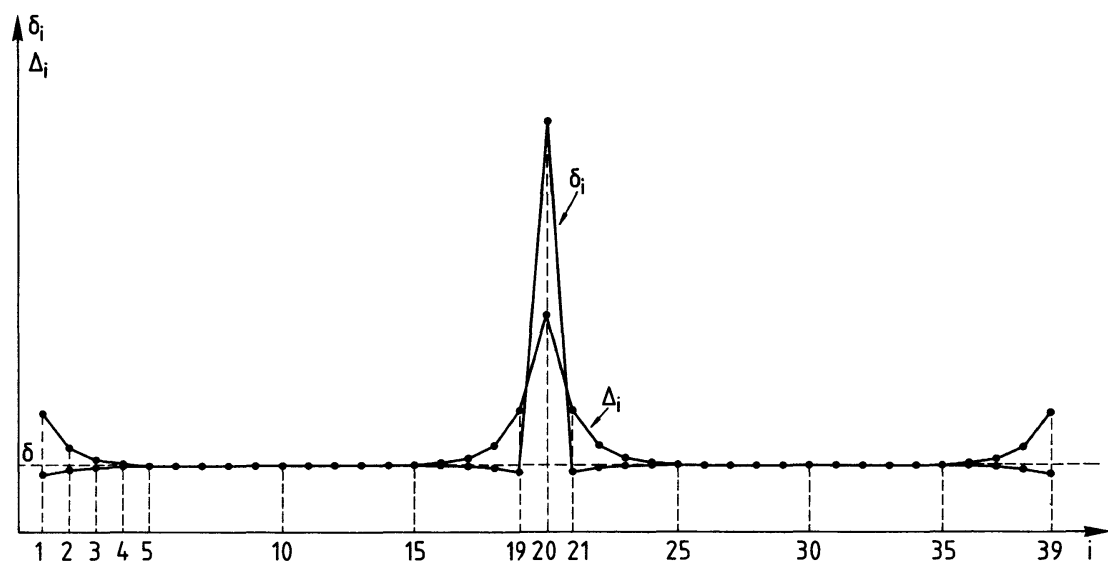

Fig. 9. - Cas d'un pincement à 20 molécules $(b=1,6)$. On a figuré ici deux périodes. La droite horizontale pointillée correspond à la valeur de $\delta$ pour la solution banale.

[Pinched structure with 20 molecules $(b=1.6)$. Two periods are represented here. The horizontal dashed line gives the value of $\delta$ for the trivial solution.]

L'application de cette méthode à notre problème entraîne une discrétisation qui devrait en principe porter sur les $x_{i}$ et $y_{i}$. Pour gagner en temps de calcul nous avons discrétisé uniquement les $\delta_{i}$ et fixé les $\Delta_{i}$ à leurs valeurs d'équilibre : $\Delta_{i}=b-\frac{1}{A} U^{\prime}\left(\delta_{i}\right)$. Un certain nombre d'essais faits avec des discrétisations de plus en plus fines n'a pas permis de trouver de nouvelles solutions.

\section{Conclusion.}

En résumé, on trouve, pour une plage de valeurs de $b$, distance naturelle entre chaînes, des pincements dans l'état fondamental de la chaîne. Il s'agit de structures périodiques où se répètent des motifs à $N=2,3, \ldots$ molécules. La période est commensurable avec la distance moyenne entre les plateaux ou avec le pas du réseau sous-jacent constitué par la discrétisation. On peut ainsi définir pour chaque type de pincement un vecteur d'onde $k \sim \frac{1}{N}$.

Les conclusions de cette étude diffèrent sensiblement des résultats de l'analyse du modèle dans la limite continue donnée par de Gennes. Ceci n'est pas étonnant lorsqu'on considère la forme typique des pincements de la figure 9. Le raccord entre deux pincements se fait par une paroi qui ne s'étend que sur un nombre très limité d'atomes (pour l'essentiel sur 3 atomes) et ne pourra donc être décrite dans la limite continue. L'amplitude de cette paroi liée au caractère discret du modèle diminue lorsque l'amplitude du potentiel $U$ diminue. Malheureusement la zone d'existence des pincements rétrécit en même temps !

La simplicité des états que nous avons observés est en contradiction, d'une part avec les phases incommensurables évoquées par de Gennes, d'autre part avec d'éventuelles phases " quasi chaotiques » auxquelles on aurait pu éventuellement s'attendre du fait de l'introduction d'un potentiel d'interaction non convexe entre plateaux [4]. Il est possible que ces phases existent sans que nous ayons su les trouver, soit du fait des méthodes employées, soit en raison d'un mauvais choix des valeurs des paramètres, mais il nous semble plutôt que ce résultat soit dû au fait que la frustration liée à la compétition entre les deux échelles de longueur du problème est beaucoup moins forte que dans des modèles similaires, comme celui de Frenkel' et Kontorova [4, 5]. 


\section{Remerciements.}

Nous remercions A. M. Levelut, P. G. de Gennes et A. Skoulios pour des conservations enrichissantes et $\mathrm{S}$. Aubry pour son intérêt tout au long du travail.

\section{Bibliographie}

[1] Guillon, D., Skoulios, A., Piechocki, C., Simon, J., Weber, P., Mol. Cryst. Liq. Cryst. 100 (1983) 275.

[2] On trouvera d'autres indications sur l'ordre dans les colonnes dans :

Levelut, A. M., J. Chim. Phys. 80 (1983) 149, et dans :

Safinya, C. et al., Phys. Rev. Lett. 53 (1984) 1172.

[3] De Gennes, P. G., J. Physique Lett. 44 (1983) L-657.

[4] Aubry, S., J. Physique 44 (1983) 147.

[5] Kontorova, T. A., Frenkel', Y. I., Zh. Eksp. Teor. Fiz. 8 (1938) 89. 